\title{
European Multicentre Tics in Children Studies (EMTICS): protocol for two cohort studies to assess risk factors for tic onset and exacerbation in children and adolescents
}

\author{
Anette Schrag ${ }^{1}$ - Davide Martino ${ }^{2} \cdot$ Alan Apter $^{3}$. Juliane Ball ${ }^{4}$ Erika Bartolini ${ }^{5} \cdot$ Noa Benaroya-Milshtein $^{3}$. \\ Maura Buttiglione $^{6} \cdot$ Francesco Cardona $^{7} \cdot$ Roberta Creti $^{8} \cdot$ Androulla Efstratiou $^{9} \cdot$ Maria Gariup $^{10,11}$. \\ Marianthi Georgitsi ${ }^{12,13} \cdot$ Tammy Hedderly $^{14}$ • Isobel Heyman ${ }^{15} \cdot$ Immaculada Margarit $^{5}$. Pablo Mir $^{16}$. \\ Natalie Moll ${ }^{17}$. Astrid Morer ${ }^{18,19,20}$. Norbert Müller ${ }^{21,22} \cdot$ Kirsten Müller-Vahl $^{23}$ - Alexander Münchau ${ }^{24}$. \\ Graziella Orefici $^{8} \cdot$ Kerstin J. Plessen ${ }^{25,26} \cdot$ Cesare Porcelli2 $^{27}$. Peristera Paschou ${ }^{28} \cdot$ Renata Rizzo $^{29} \cdot$ Veit Roessner $^{30}$. \\ Markus J. Schwarz ${ }^{17} \cdot$ Tamar Steinberg $^{3} \cdot$ Friederike Tagwerker Gloor $^{4} \cdot$ Zsanett Tarnok $^{31} \cdot$ Susanne Walitza $^{4}$. \\ Andrea Dietrich $^{32}$. Pieter J. Hoekstra ${ }^{32}$. EMTICS Collaborative Group
}

Received: 21 January 2018 / Accepted: 28 June 2018 / Published online: 7 July 2018

(c) The Author(s) 2018

\begin{abstract}
Genetic predisposition, autoimmunity and environmental factors [e.g. pre- and perinatal difficulties, Group A Streptococcal (GAS) and other infections, stress-inducing events] might interact to create a neurobiological vulnerability to the development of tics and associated behaviours. However, the existing evidence for this relies primarily on small prospective or larger retrospective population-based studies, and is therefore still inconclusive. This article describes the design and methodology of the EMTICS study, a longitudinal observational European multicentre study involving 16 clinical centres, with the following objectives: (1) to investigate the association of environmental factors (GAS exposure and psychosocial stress, primarily) with the onset and course of tics and/or obsessive-compulsive symptoms through the prospective observation of at-risk individuals (ONSET cohort: 260 children aged 3-10 years who are tic-free at study entry and have a first-degree relative with a chronic tic disorder) and affected individuals (COURSE cohort: 715 youth aged 3-16 years with a tic disorder); (2) to characterise the immune response to microbial antigens and the host's immune response regulation in association with onset and exacerbations of tics; (3) to increase knowledge of the human gene pathways influencing the pathogenesis of tic disorders; and (4) to develop prediction models for the risk of onset and exacerbations of tic disorders. The EMTICS study is, to our knowledge, the largest prospective cohort assessment of the contribution of different genetic and environmental factors to the risk of developing tics in putatively predisposed individuals and to the risk of exacerbating tics in young individuals with chronic tic disorders.
\end{abstract}

Keywords Genetics $\cdot$ Longitudinal $\cdot$ Obsessive-compulsive disorder $\cdot$ Stress $\cdot$ Streptococcal infection $\cdot$ Tourette syndrome

The EMTICS group members are listed in Appendix section.

Electronic supplementary material The online version of this article (https://doi.org/10.1007/s00787-018-1190-4) contains supplementary material, which is available to authorized users.

Anette Schrag and Davide Martino joint first authors.

Pieter J. Hoekstra

p.hoekstra@accare.nl

Extended author information available on the last page of the article

\section{Introduction}

Tic disorders are common, childhood-onset neuropsychiatric conditions characterised by the presence of sudden, rapid, recurrent, non-rhythmic motor movements (motor tics) and/ or vocalisations (vocal tics). Tic disorders are diagnosed when motor tics and vocal tics, either alone (chronic motor or vocal tic disorder) or in combination [Tourette syndrome (TS), manifesting with multiple motor tics and at least one vocal tic], begin before age 18 and last more than 1 year, in the absence of tics being attributable to a substance or another medical condition [1]. The prevalence of tics 
during childhood/adolescence is close to $3 \%$, and that of TS is approximately $0.8 \%$ between the ages of 6 and 18 years [2-4]. Tics and their associated neuropsychiatric comorbidities [attention-deficit/hyperactivity disorder (ADHD), obsessive-compulsive disorder (OCD), anxiety and depressive disorders, autism spectrum disorders] often affect quality of life of patients and families, as well as social and academic functioning of patients [5-9].

Our knowledge of the pathophysiological mechanisms involved in TS and other chronic tic disorders is still limited. Pharmacological and behavioural treatment options for TS represent the mainstay of treatment for tics, but both have limitations related to patients' access to care, tolerability, and efficacy. In addition, at least $5 \%$ of patients with TS attending specialized clinics may have a very severe form that is refractory to non-invasive treatments [10]. There is still major need for new treatments and effective preventative methods, potentially fostered by a better understanding of disease pathophysiology.

TS is viewed as a complex neuropsychiatric disorder, which is likely to be related to an as yet poorly understood interaction between genetic and environmental susceptibility factors. While the heritability of TS has been estimated to be as high as 0.77 in a large scale multigenerational family study [11], a recent twin-family study found much lower heritability estimates, ranging between 0.25 and 0.37 [12], indicating a substantial role for environmental factors. The complex trait of tic disorders is polygenic, similar to most psychiatric disorders. Over the past decade, genetic factors associated with TS have been explored primarily through genome-wide approaches including genome-wide association studies (GWASs), analysis of copy number variants (CNVs), and whole exome sequencing (WES) approaches. GWASs in TS have to date failed to identify highly genomewide significant loci $[13,14]$, likely due also to limited sample sizes, which were smaller than in other major psychiatric GWASs [15]. The contribution of rare structural variation to the genetic architecture of TS is supported by recent analyses of rare CNVs, which indicate that approximately $1 \%$ of TS cases carry one of these CNVs, highlighting also genomewide significant loci increasing TS risk, i.e. NRXN1 deletions and CNTN6 duplications [16]. Like GWASs, WES studies in TS are also limited by their small sample sizes compared to other complex psychiatric traits; an association with de novo damaging variants has been reported for a dozen candidate genes [17-20] and needs to be confirmed by studies with larger sample sizes. There is also a striking paucity of gene expression studies in tic disorders. Studies in this area focused on biological pathways related to neurotransmitters and immune regulation [17, 21-23], but were based on small sample sizes, did not clarify whether the observed changes were causes or consequences of the behavioural phenotype, and were never adequately combined to genomic data. Finally, the exploration of epigenetic modifications associated with TS and other chronic tic disorders is still in its dawning [12, 24].

Some pre- and perinatal factors potentially interfering with normal brain development have been explored also in association with TS, although the related evidence differs for quality and methodology used across the different variables explored [e.g. 25-29]. During the past decade, a limited number of studies have also explored the contribution of psychosocial stress with overall inconclusive results, even if clinical experience does seem to support a role of stress in patients with TS [30]. A prospective evaluation based on questionnaires suggested an effect of psychosocial stress as a short-term predictor of tic severity [31]. Another study showed increased cortisol responses during acute stressors [32], but this was not confirmed by Buse et al. [33], who observed the opposite acute effect on a behavioural acute stress test (Trier Social Stress Test), coupled to salivary cortisol determination and autonomic measures of the stress response. Obvious limitations of this literature are the limited sample sizes and the lack of longitudinal data exploring biological markers of acute and chronic stress, as well as direct measures of hypothalamic-pituitary-adrenal (HPA) axis activation.

An important research area, relevant to environmental influences in relation to tic disorders, is the involvement of abnormal innate and adaptive immune responses in the pathogenesis of tics and related behavioural symptoms [34]. A dysfunctional neural-immune cross-talk has been observed in patients with TS, in analogy to other neurodevelopmental disorders (e.g. autism spectrum disorder). Recent data from prospective population-based cohorts have demonstrated a 30\% increased incidence rate of TS in male offspring of women with an autoimmune disease [34], and a higher risk of any autoimmune disease among first-degree relatives of patients with OCD and chronic tic disorders [35]. Clinical studies have documented increased proliferation and activation of $\mathrm{B}$ and $\mathrm{T}_{\mathrm{H} 1}$ lymphocytes, increased proinflammatory cytokine levels, a decreased number of $T_{\mathrm{REG}}$ lymphocytes, dysregulated immunoglobulin synthesis, supporting the existence of adaptive immune responses skewed towards an inflammatory state TS [36, 37]. Furthermore, both post mortem data [38] and in vivo molecular imaging [39] suggest microglial activation in TS. Microglia cells are key players of the immune system in the central nervous system and play an increasingly recognized role in brain infections, neuroinflammation and neurodegeneration [40].

Alongside stress, infectious pathogens are obvious potential culprits for the overactivity of immune responses documented in tic disorders. A specific interest in a role for common infections (pharyngotonsillitis) caused by group A streptococcus (GAS or Streptococcus pyogenes) has been drawn by the description in 1998 of Paediatric Autoimmune 
Neuropsychiatric Disorders Associated with Streptococcal Infections (PANDAS), a putatively autoimmune syndrome manifesting with obsessive-compulsive symptoms, tics, emotional lability, anxiety and regressive behaviour triggered by this pathogen [41, 42]. In 2012, the broader syndromic entity of Pediatric Acute Neuropsychiatric Syndromes (PANS) has been proposed, which encompasses PANDAS but includes other possible aetiologies [43]. Both PANS and PANDAS are viewed as a subtype of paediatric OCD and/or TS that present with an abrupt onset or exacerbation of neuropsychiatric symptoms [44]. An autoimmune mechanism triggered by molecular mimicry between GAS bacterial and host neural antigens has been proposed for PANDAS, and putatively pathogenic biomarkers, e.g. autoantibodies directed against dopamine D2 receptors and antibody-mediated calcium/calmodulin-kinase II activation in cell-based assays, have been reported and included in a proposed diagnostic panel (the Cunningham panel) $[45,46]$. The diagnostic accuracy of this panel is, however, still discussed [47], and the potential usefulness of these biomarkers in highlighting an 'autoimmune' subgroup of patients with tic disorders is unexplored. More recent work has highlighted the rationale of exploring immune regulatory markers in this group of patients, and decreased systemic levels of vitamin D have been documented in an Italian cohort of patients with PANDAS [48]. Preliminary evidence reported the presence of anti-D2 dopamine receptor antibodies in a small proportion of individuals with TS Dale [49], supporting the rationale for further exploration of autoantibody markers in this condition.

Although tics are now considered an associated rather than a core feature of PANDAS and PANS [42], researchers have tried to address, during the past decade, the still unanswered question of whether and how infections, and above all GAS infections, are associated with the onset and/ or the exacerbation of tics and related behavioural symptoms. Large, retrospective, population-based cohort studies have provided some degree of evidence that GAS infections may be associated with the onset of tics [50-53] although discrepancies across studies exist [54]. At the same time, smaller, prospective studies have failed to show a clear association between GAS infections and tic exacerbations [55-59]. Nevertheless, one of these prospective studies reported a multiplicative interaction between GAS infections and psychosocial stress in predicting tic and obsessive-compulsive symptom severity in the short term [60]. Overall, these previous studies are limited by small sample sizes and some ambiguities in design (addressed in more depth in our "Discussion"), which justify the conduction of larger, more ambitious, prospective observations that take into account the complexity of the clinical phenomenology of tic disorders, the genetic diversity of GAS, and the high inter-individual variability of GAS-induced immune responses [61]. An adequately sized clinic-based prospective study that collects behavioural, microbiological, immuneendocrinological, genomic and transcriptomic data, provides a unique opportunity to advance the field by tackling some of the unanswered questions on the aetiopathogenesis of chronic tic disorders. The objectives and design of the multi-centre pan-European collaborative study EMTICS, funded by the European Commission seventh Framework programme (FP7), were conceived to address several of the knowledge gaps summarized above.

\section{Core objectives of EMTICS}

1. To investigate the association between putatively relevant environmental factors, genome-wide genetic factors, and gene expression patterns upon the risk of developing clinically relevant exacerbations of tics and/ or OCD symptoms in youth with an established chronic tic disorder (COURSE cohort).

2. To investigate the association between putatively relevant environmental factors, genome-wide genetic factors, and gene expression patterns upon the risk of new onset of tics in children who are first-degree relatives of patients with an established chronic tic disorder (ONSET cohort).

For the first and second objectives, the explored environmental exposures comprise: recent GAS infection, GAS carriage status, other recent infections, fluctuations of psychosocial stress, cortisol as a marker of chronic stress and pre- and perinatal adversities.

3. To characterise patterns of the host innate and adaptive immune responses that are associated with clinical events of interest, i.e. onset of tics and clinically relevant exacerbations of tics and/or OCD symptoms in the two clinical cohorts. This will comprise analyses of immune effectors (e.g. cytokines, immunoglobulins, acute phase reactants, other effector molecules including those belonging to the tryptophan/kynurenine pathway and vitamin D) and immune cell phenotyping.

4. To characterise patterns of the host antibody response to GAS and other pathogens previously reported in association with chronic tic disorders. The anti-GAS antibody patterns will be investigated with state-of-the-art microarray technology.

5. To develop multimodal prediction models for the risk of onset of tics in first-degree relatives of patients with chronic tic disorders, as well as for the risk of clinically relevant exacerbations of tics and/or OCD symptoms in youth with an established chronic tic disorder. 


\section{Methods}

\section{Participants}

Children aged 3-16 years were recruited from the sixteen child and adolescent psychiatry and paediatric neurology outpatient clinics (listed in the "Appendix"), from patients and families already known to these services or through advertisement of the study to patient organizations and other health professionals. This project was based on two separate cohort studies: ONSET and COURSE. The study was approved by the Institutional Review Boards of the participating centres. Parents and their child (ren) provided written informed consent and assent as appropriate according to ethical regulations. While travel costs of participating families were reimbursed no additional fees were paid to participants. The first participant was enrolled in EMTICS in January 2013 and the study has concluded with the last visit of the last ONSET patients in June 2018.

\section{Inclusion criteria}

For the ONSET cohort, children aged 3-10 years were recruited who are first-degree relatives (siblings or children) of patients with TS or another chronic tic disorder (criteria according to the Diagnostic and Statistical Manual fourth edition, text revision [62]), but had themselves never had tics, OCD or trichotillomania. This age range reflects the range of age at first onset of tics in chronic tic disorders, as reported by the majority of naturalistic studies on these conditions [63]. For the COURSE cohort, patients aged 3-16 years with an established diagnosis of TS or chronic motor or vocal tic disorder according to DSM IV-TR criteria [62] were recruited. More than one sibling per family could participate in both studies.

\section{Exclusion criteria}

Children unable to understand and comply with protocol, or whose parents were unable to comply, and children with serious medical/neurological illness or treated with antibiotics in the past month were excluded from both ONSET and COURSE cohorts. The presence of comorbid neuropsychiatric conditions such as autism spectrum disorder was not an exclusion criterion, nor was suspected PANS or PANDAS. Use of medications was not an exclusion criterion; however, all medication a child was taking during the study was recorded. Children who refused throat swabs, blood draws or hair strains could still participate in the studies.

\section{Study design and procedures}

\section{Longitudinal study ONSET}

The ONSET study examines the association of the new onset of tics with exposure to pharyngeal GAS carriage or infection and other environmental and genetic factors, using clinical and laboratory assessments, over a 3-year period where the onset of tics represents the event of interest. Participants were evaluated every 2 months, interchangeably by 4-monthly scheduled hospital visits and telephone interviews. Telephone interviews were scheduled in the middle of the 4 months' intervals between hospital visits (see Fig. 1 and supplementary Table 1). Irrespective of the planned visit schedule, parents were instructed to communicate any possible sign of tic onset to the study centre as soon as possible (e.g. by phone or email). To this purpose, all possible symptoms indicative of a possible onset of tics were thoroughly explained to parents at the baseline visit in ONSET.

Data collection was structured on three levels of observation: (1) through a weekly diary in which children's parents were asked to indicate symptom onset, aimed at the earliest possible detection of onset of tics throughout the whole study duration plus an inventory of signs of infectious disease (common cold), obsessive-compulsive symptoms and stressful events; (2) scheduled telephone interview once every 4 months with review of the weekly diaries since the last assessment and clinical evaluations performed by the study clinician to children's parents; and (3) visits in hospital every 4 months over the 3-year duration of the follow-up period, which comprised a more extensive clinical evaluation and collection of biological specimens (for measurements see Table 1).

If parents reported possible onset of tics outside of planned visits or telephone interviews, an "unscheduled tic onset evaluation telephone interview" was held by the study clinician to investigate whether possible onset of tics had occurred. Tic onset was defined as the first occurrence of any sudden, rapid, recurrent, non-rhythmic involuntary motor movement and/or vocalisation noticed on at least three separate days within a period of 3 weeks. If the evaluation pointed to a possible tic onset (even when somewhat unsure), an "onset of tics hospital visit" was scheduled preferably within 1 week or at the earliest opportunity for extended clinical evaluation and collection of biological material. This served to assess the range of tics and/or obsessive-compulsive symptoms, symptoms and signs of infection (through throat swabs and blood tests for immunological markers of infection).

An "onset of tics hospital visit" was also scheduled when a (not previously reported) possible onset of tics was detected during a scheduled telephone interview while reviewing parents' weekly diaries over the past 2 months 


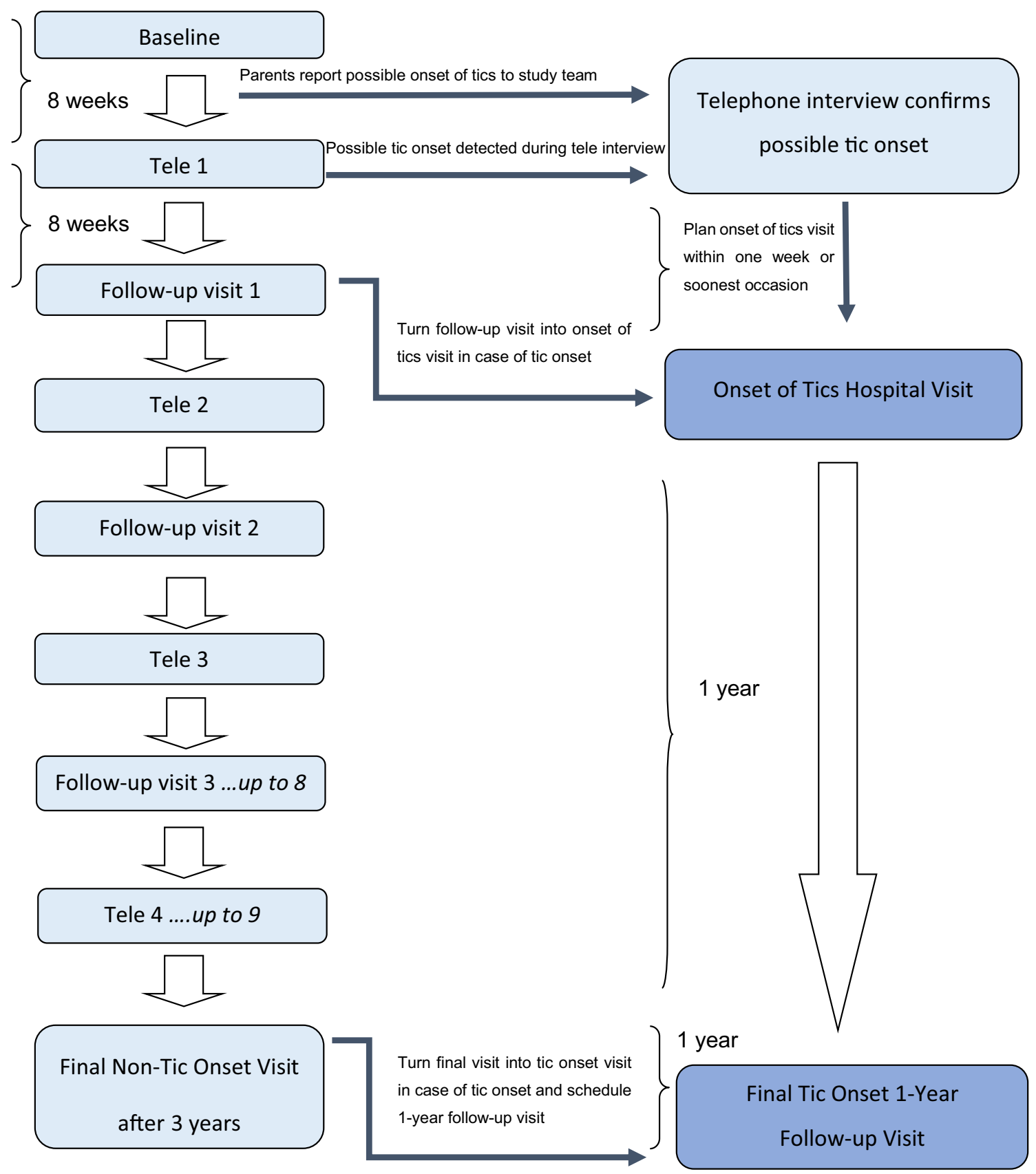

Fig. 1 ONSET study flow chart. Tele telephone interview. If no tic onset was detected and confirmed then the original assessment schedule (left side) was retained. After an onset of tics visit, all assess-

since the last assessment. Likewise, a planned follow-up hospital visit was turned into an "onset of tics hospital visit" when a possible onset of tics was confirmed during the planned visit. If tic onset occurred, all further planned assessments were discarded whereas a final follow-up visit was scheduled 1 year later that consisted of a clinical evaluation to confirm the true onset and type of tic disorder (according to DSM-IV-TR criteria) [62]. If an onset of tics was definitely not confirmed (e.g. tic-like phenomena could ments were discarded, except for a 1-year follow-up visit. The minimum study period was 1 year, tic onset at the final visit increased the maximum study period to 4 years

be better explained as symptoms of allergy or infectious disease), then the original visit schedule was retained. The maximum duration of the follow-up period was 3 years from enrolment if there was no tic onset (concluding with a "final non-tic onset visit"; a maximum of ten planned hospital visits and nine telephone interviews took place; see supplemental Table 1), and a maximum of 4 years from enrolment if tics developed (concluding with a "final tic onset 1-year follow-up visit", see Fig. 1). 
Table 1 Summary of clinical and laboratory measurements at ONSET visits

\begin{tabular}{|c|c|c|c|c|c|}
\hline & Baseline visit & $\begin{array}{l}\text { 4-monthly } \\
\text { follow-up visit }\end{array}$ & Tic onset visit & $\begin{array}{l}\text { Final visit 1-year } \\
\text { post-tic onset }^{\mathrm{a}}\end{array}$ & $\begin{array}{l}\text { Final visit } \\
\text { without tic } \\
\text { onset }\end{array}$ \\
\hline Demographics and family medical history & $\checkmark$ & & & & \\
\hline Child's medical history & $\checkmark$ & & & $\checkmark$ (update) & $\checkmark$ (update) \\
\hline Inventory of infections & $\checkmark$ & $\vee^{\mathrm{b}}$ & $\checkmark$ & & $\checkmark$ \\
\hline Psychotropic drug checklist & $\checkmark$ & $\checkmark$ & $\checkmark$ & $\checkmark$ & $\checkmark$ \\
\hline Prenatal, perinatal and developmental history & $\checkmark$ & & & & \\
\hline Exploration of possible tic onset & & $\vee^{\mathrm{b}}$ & & & $\checkmark$ \\
\hline YGTSS & & & $\checkmark$ & $\checkmark$ & \\
\hline PUTS & & & $\checkmark$ & $\checkmark$ & \\
\hline CY-BOCS & $\checkmark$ & $\checkmark$ & $\checkmark$ & $\checkmark$ & $\checkmark$ \\
\hline CGI-S (tics) & & & $\checkmark$ & $\checkmark$ & \\
\hline CGI-S (overall) & $\checkmark$ & $\checkmark$ & $\checkmark$ & $\checkmark$ & $\checkmark$ \\
\hline CGI-I (overall) & & $\checkmark$ & $\checkmark$ & $\checkmark$ & $\checkmark$ \\
\hline Tic disorder diagnosis & & & & $\checkmark$ & \\
\hline OCD diagnosis & & & & $\checkmark$ & $\checkmark$ \\
\hline ADHD diagnosis & $\checkmark$ & & & $\checkmark$ & $\checkmark$ \\
\hline Trichotillomania diagnosis & & & & $\checkmark$ & $\checkmark$ \\
\hline SDQ & $\checkmark$ & $\checkmark$ & $\checkmark$ & $\checkmark$ & $\checkmark$ \\
\hline SNAP-IV & $\checkmark$ & $\checkmark$ & $\checkmark$ & $\checkmark$ & $\checkmark$ \\
\hline ASSQ & $\checkmark$ & & & & \\
\hline Kindl-R & $\checkmark$ & $\checkmark$ & $\checkmark$ & $\checkmark$ & $\checkmark$ \\
\hline PSS-P-10; PSS-C-10 ( $\geq 11$ years) & $\vee^{c}$ & $\checkmark^{\mathrm{d}}$ & $\checkmark$ & & $\checkmark$ \\
\hline Inventory of stressful events & $\checkmark$ & $\vee^{\mathrm{b}}$ & $\checkmark$ & & $\checkmark$ \\
\hline Throat swab & $\checkmark$ & $\vee^{e}$ & $\checkmark$ & & $\checkmark$ \\
\hline Serum sample for immune analyses ${ }^{f}$ & $\checkmark$ & $\sqrt{ }^{g}$ & $\checkmark$ & & $\checkmark$ \\
\hline Fresh blood sample & $\checkmark$ & & $\checkmark$ & & \\
\hline Blood sample for DNA & & & $\checkmark$ & & \\
\hline Blood sample for RNA & $\checkmark$ & & $\checkmark$ & & \\
\hline Hair sample for cortisol analysis & $\checkmark$ & $\checkmark$ & $\checkmark$ & & $\checkmark$ \\
\hline
\end{tabular}

See the main text for a description of measures

${ }^{a}$ The purpose of this visit was to establish whether the onset of tics was indeed indicative of the onset of a chronic tic disorder and to assess the possible presence of comorbidity

${ }^{\mathrm{b}}$ Also assessed during 4-monthly telephone interviews

${ }^{\mathrm{c}}$ No PSS-C-10

${ }^{\mathrm{d}}$ PSS-P-4 during 4-monthly telephone interviews

e Only for follow-up visits \#3 and \#6

${ }^{\mathrm{f}}$ Antibody responses to Group A streptococcal infections and other infectious pathogens including Anti-streptolysin O (ASO) and anti-deoxyribonuclease B antibody titres (anti-DNAse B), cytokines, inflammatory status (C-reactive protein), and autoantibodies

${ }^{\mathrm{g}} \mathrm{ASO}$ and anti-DNAse B measurements only

${ }^{\mathrm{h}}$ For immune response analyses only in a sub-sample of participants

\section{Longitudinal study COURSE}

The COURSE study is a cohort study that prospectively observed over a maximum period of 18 months 715 children and adolescents with an age range of 3-16 years affected by a tic disorder [62], where exacerbations of tics represent the events of interest. Data collection was analogous to the ONSET study, with interchanging 4-monthly planned hospital visits and telephone interviews as well as parental weekly diaries aimed at identifying tic exacerbations (see Fig. 2 and supplementary Tables 2 and 3).

Similar to the ONSET protocol, parents were asked to report any noticeable increase in tic severity, seemingly unrelated to a reduction or discontinuation of prescribed medication, to the study team. If the study clinician subsequently through an unscheduled telephone interview 


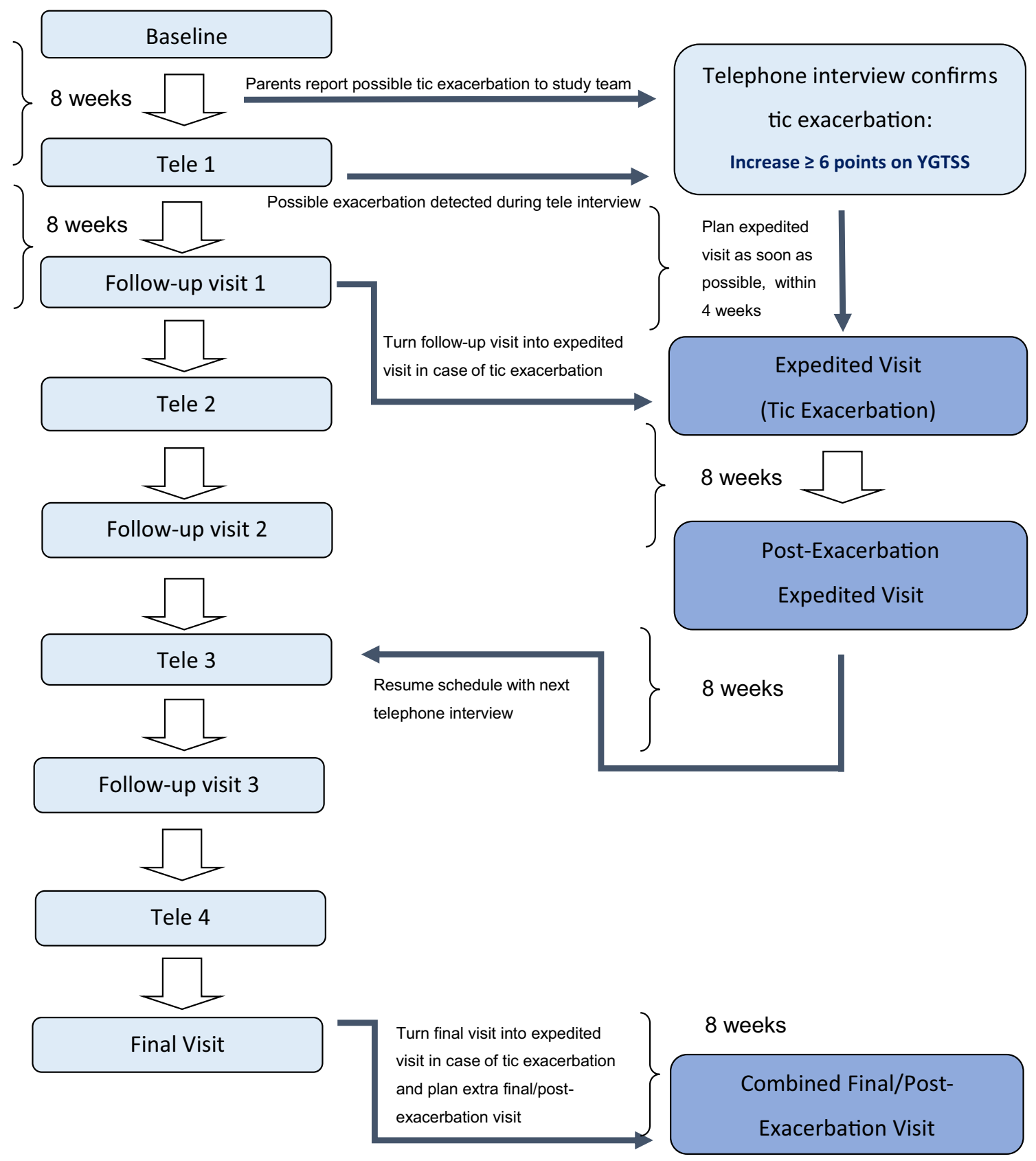

Fig. 2 COURSE study flow chart. Tele telephone interview, YGTSS Yale Global Tic Severity Scale. If no tic exacerbation was detected and confirmed then the original 16 month assessment schedule (left side) was retained. The maximum study period was 18 months if a tic

confirmed a tic exacerbation, defined as an increase of at least six points on the total tic severity score (i.e. sum of motor and vocal tic severity, but not impairment) of the Yale Global tic severity scale (YGTSS) [64] compared to the score during the previous assessment, an appointment for an expedited visit was scheduled preferably within 1 week, but no more than within 4 weeks. This expedited visit was followed by a "post-exacerbation visit" 2 months later, exacerbation was detected at the final visit. A maximum of two pairs of expedited and post-exacerbation visits was possible. Expedited visits shortened the study period accordingly

aimed at capturing tic remission, defined as a subsequent decrease of at least six points on the total tic severity score. Thereafter, a bi-monthly schedule of either a telephone interview or hospital visit was resumed. In COURSE, the total number of hospital visits was five with four planned telephone interviews, or six visits when an exacerbation had occurred at the final visit; a maximum number of two pairs of exacerbation plus post-exacerbation visits were possible 
(see supplementary Table 3 for the different measurement scenarios).

An exacerbation of tics (not previously reported by parents) may also have been detected during the bi-monthly assessments either through telephone or hospital visit while reviewing the weekly diaries. While preferably the exacerbation should then still be ongoing it was allowed to schedule an expedited visit within 4 weeks of the tic exacerbation. Thus, it may have occurred that tic severity was already in remission at the time of the expedited visit. If a tic exacerbation came under the attention during a planned follow-up visit, then the visit was turned into an "expedited tic exacerbation visit". Finally, in some of the participating clinical sites, COURSE participants were considered for inclusion in a separate antibiotic treatment study if their microbiological testing revealed a positive culture for GAS.

\section{Missed visits and end of study}

Planned follow-up hospital visits and telephone interviews as well as "post-exacerbation visits" could be scheduled 2 weeks earlier or later than according to the visit schedule (see supplementary Tables 1 and 2; except "tic onset visits" that could be scheduled at the earliest opportunity, or "expedited tic exacerbation visits" and "final visits" that could be scheduled with a delay of up to 4 weeks). If this was not possible, the visit or interview was considered missed. Three consecutive missed appointments (visits or telephone interviews) led to study discontinuation. Children not keeping weekly diaries could still continue to participate in the study.

Study end is the date of the last visit of the last participant; for the ONSET study this is after 3 years after baseline in case of no tic onset; or 1 year after onset of tics with a maximum of 4 years. For the COURSE study, this is after 16 months or less if there were tic exacerbations, and a maximum of 18 months when a tic exacerbation was identified during the planned final visit.

\section{Clinical and laboratory measures}

\section{Clinical measures}

Tables 1, 2, 3 present a summary of clinical and laboratory measurements at ONSET and COURSE visits, respectively. The main outcome measure for both cohort studies is based on the YGTSS, the gold standard instrument to rate tic severity, which has robust psychometric properties [64]. Another main, well-validated measure was the Children's Yale Brown Obsessive-Compulsive Scale (CY-BOCS; [65]), used to rate obsessive-compulsive symptom severity. Study clinicians well-experienced in the evaluation and treatment of tic disorders and associated conditions used these semistructured interviews to assess past week symptom severity and the current and past presence of tic disorders, OCD, and attention-deficit/hyperactivity disorder (ADHD) according to DSM-IV-TR criteria [62]. Clinicians also rated the Clinical Global Impression Scale Severity for overall functioning and/or tics (CGI-S for severity and CGI-I for improvement) [66] and checked a list of psychotropic medication use during the past 2 weeks.

A set of parent-reported questionnaires was used to assess participants' demographics, the child's medical history (e.g. immune-related conditions such as allergic rhinitis or atopic dermatitis), family medical history (psychiatric, neurologic and autoimmune diseases) [66], prenatal, perinatal and developmental history (Modified Schedule for Risk and Protective factors Early in Development, MSRPFED) capturing 38 possible adverse situations [28, 67, 68] and degree of child experienced psychosocial stress over the previous month by the Perceived Stress Scale (PSS-P-10 at hospital visits; PSS-P-4 in telephone interviews) [69]. Parent ratings of child's psychopathological symptoms included the Strengths and Difficulties Questionnaire (SDQ) [70, 71], the Swanson, Nolan and Pelham-version IV rating scale (SNAPIV) $[72,73]$ to assess ADHD severity, and the Autism Spectrum Screening Questionnaire (ASSQ) [74]. Finally, the Kindl-R questionnaire (4-7 and 8-16 years parent versions) [75] measured health-related impact on the quality of life.

Child reports were the Premonitory Urge for Tics Scale (PUTS) [76], which measures unpleasant sensorimotor phenomena that often precede tics and the self-reported Perceived Stress Scale (PSS-C-10) [69]) in children $\geq 11$ years. The weekly diaries covered an inventory of possible infections (i.e. symptoms of common cold such as running nose, persistent cough or fever) and related diagnosis and treatment, possible medication changes, occurrence of stressful events, and possible onset of tics or fluctuations (especially exacerbations) in tics and OCD symptoms.

\section{Laboratory measures}

See Table 3 for an overview of laboratory measures. The main microbiological measures were GAS colonisation by throat swabbing and processing using a consensus-defined methodology (pour plate method) [55, 77]. The microbiological typing of bacterial GAS population was performed by emm typing, Multiple Locus Variable number of tandem repeats Analysis (MLVA) and Multi Locus Sequence Typing (MLST). Exposure to GAS in study participants was investigated by measuring anti-streptolysin O titre (ASOT) and anti-DNAseB antibody titre. Moreover, protein microarray technology described elsewhere by one of the teams participating in the EMTICS consortium [61] was used to compare the patterns of antibody responses to a panel of GAS antigens in ONSET participants who have or have not developed tics. By the same approach, antibody profiles 
Table 2 Summary of clinical and laboratory measurements at COURSE visits

\begin{tabular}{|c|c|c|c|c|c|}
\hline & Baseline visit & $\begin{array}{l}\text { 4-monthly } \\
\text { follow-up visit }\end{array}$ & $\begin{array}{l}\text { Expedited visit in case } \\
\text { of tic exacerbation }\end{array}$ & $\begin{array}{l}\text { Post-exacerba- } \\
\text { tion visit }{ }^{\mathrm{a}}\end{array}$ & Final visit \\
\hline Demographics and family medical history & $\checkmark$ & & & & \\
\hline Child's medical history & $\checkmark$ & & & & $\checkmark$ (update) \\
\hline Inventory of infections & $\checkmark$ & $\checkmark^{\mathrm{b}}$ & $\checkmark$ & $\checkmark$ & $\checkmark$ \\
\hline Psychotropic drug checklist & $\checkmark$ & $\checkmark$ & $\checkmark$ & $\checkmark$ & $\checkmark$ \\
\hline Prenatal, perinatal and developmental history & $\checkmark$ & & & & \\
\hline Evaluation of possible tic exacerbation & & $\checkmark^{\mathrm{b}}$ & & & $\checkmark$ \\
\hline YGTSS & $\checkmark$ & $\vee^{\mathrm{b}}$ & $\checkmark$ & $\checkmark$ & $\checkmark$ \\
\hline PUTS & $\checkmark$ & $\checkmark$ & $\checkmark$ & $\checkmark$ & $\checkmark$ \\
\hline CY-BOCS & $\checkmark$ & $\vee^{\mathrm{b}}$ & $\checkmark$ & $\checkmark$ & $\checkmark$ \\
\hline CGI-S (overall and tics) & $\checkmark$ & $\checkmark$ & $\checkmark$ & $\checkmark$ & $\checkmark$ \\
\hline CGI-I (overall and tics) & & $\checkmark$ & $\checkmark$ & $\checkmark$ & $\checkmark$ \\
\hline Tic disorder diagnosis & $\checkmark$ & & & & $\checkmark$ \\
\hline OCD diagnosis & $\checkmark$ & & & & $\checkmark$ \\
\hline ADHD diagnosis & $\checkmark$ & & & & $\checkmark$ \\
\hline Trichotillomania diagnosis & $\checkmark$ & & & & $\checkmark$ \\
\hline SDQ & $\checkmark$ & $\checkmark$ & $\checkmark$ & $\checkmark$ & $\checkmark$ \\
\hline SNAP-IV & $\checkmark$ & $\checkmark$ & $\checkmark$ & $\checkmark$ & $\checkmark$ \\
\hline ASSQ & $\checkmark$ & & & & \\
\hline Kindl-R & $\checkmark$ & $\checkmark$ & $\checkmark$ & $\checkmark$ & $\checkmark$ \\
\hline PSS-P-10; PSS-C-10 ( $\geq 11$ years) & $\checkmark$ & $\vee^{c}$ & $\checkmark$ & $\checkmark$ & $\checkmark$ \\
\hline Inventory of stressful events & $\checkmark$ & $\vee^{b}$ & $\checkmark$ & $\checkmark$ & $\checkmark$ \\
\hline Throat swab & $\checkmark$ & $\checkmark$ & $\checkmark$ & $\checkmark$ & $\checkmark$ \\
\hline Serum sample for immune analyses ${ }^{\mathrm{d}}$ & $\checkmark$ & $\vee^{\mathrm{e}}$ & $\checkmark$ & $\checkmark$ & $\checkmark$ \\
\hline Fresh blood sample ${ }^{\mathrm{f}}$ & & & $\checkmark$ & $\checkmark$ & \\
\hline Blood sample for DNA & $\checkmark$ & & & & \\
\hline Blood sample for RNA & & & $\checkmark$ & $\checkmark$ & \\
\hline Hair sample for cortisol analysis & $\checkmark$ & $\checkmark$ & $\checkmark$ & $\checkmark$ & $\checkmark$ \\
\hline
\end{tabular}

See the main text for a description of measures

${ }^{\mathrm{a}}$ Two months after the expedited visit to capture possible tic remission

${ }^{\mathrm{b}}$ Also assessed during 4-monthly telephone interviews

${ }^{\mathrm{c}}$ PSS-P-4 during 4-monthly telephone interviews

${ }^{\mathrm{d}}$ Antibody responses to Group A streptococcal infections and other infectious pathogens including anti-streptolysin O (ASO) and anti-deoxyribonuclease B antibody titres (anti-DNAse B), cytokines, inflammatory status (C-reactive proteins), and autoantibodies

${ }^{\mathrm{e}} \mathrm{ASO}$ and anti-DNAse B measurements only

${ }^{\mathrm{f}}$ For immune response analyses only in a sub-sample of participants

in the COURSE participants were compared pre and post exacerbation.

Other performed serum immune measurements included measurement of IgA-, IgM- and IgG-titres against Mycoplasma pneumoniae, Chlamydia spp., Epstein-Barr virus (EBV), Borrelia burgdorferi, and Toxoplasma gondii; SCD14, interleukin-6, interleukin-17, tumor necrosis factor (TNF)- $\alpha$, TNF-RI, TNF-RII, immunoglobulin (IgA, IgM, $\mathrm{IgG})$ subclasses, and ratio between pentameric and monomeric CRP to examine the possibility of impaired resolution of inflammation; tryptophan, kynurenine, kynurenic acid, 3-hydroxykynurenine, xanthurenic acid, anthranilic acid, 3-hydroxyanthranilic acid, quinolinic acid, picolinic acid, 5-hydroxyindoleacetic acid and other important intermediates of the tryptophan pathway. Furthermore, the vitamin D status was measured and in a small sub-sample, immune cell-based measures include phenotyping for the main innate and adaptive immunological cell types, including $\mathrm{T}$ cells (CD3, CD4, CD8), B cells (CD19) and natural killer cells (CD56+). Functional analysis assessed the production of inflammatory cytokines post-stimulation/activation by bacterial pathogen triggers. Autoantibodies measured in serum, by cell-based assay [49] included anti-neuronal antibodies targeting candidate self-antigens for post-streptococcal 
Table 3 Laboratory measures

\begin{tabular}{|c|c|c|}
\hline Analysis & Material & Laboratory parameters and laboratories \\
\hline GAS colonisation & Throat swabs & $\begin{array}{l}\text { Bacterial group A streptococcal (GAS) population at each indi- } \\
\text { vidual centre }\end{array}$ \\
\hline Anti-streptococcal antibody titres & Serum & $\begin{array}{l}\text { Anti-streptolysin O (ASO), Anti-desoxyribonuclease B (anti- } \\
\text { DNAseB) at LMU }\end{array}$ \\
\hline Antibodies to non-streptococcal pathogens & Serum & $\begin{array}{l}\text { Mycoplasma pneumoniae (Myco_IgG), Chlamydia trachomatis } \\
\text { (Chlamy_IgG), Epstein-Barr virus (EBV), Borrelia burgdorferi } \\
\text { (Borrel_IgG), and Toxoplasma gondii (Toxo_IgG) at LMU }\end{array}$ \\
\hline Anti-streptococcal immune response & Serum & Antibody responses to GAS multiple antigens at GSK \\
\hline Autoantibodies by cell-based assay & Serum & $\begin{array}{l}\text { Anti-neuronal antibodies targeting candidate self-antigens for post- } \\
\text { streptococcal neuropsychiatric disorders, mainly dopamine D2 } \\
\text { receptors at Bari }\end{array}$ \\
\hline Cytokine receptors and immunoglobulins & Serum & $\begin{array}{l}\text { Interleukin-6 (IL-6), interleukin-17F (IL-17F), tumor necrosis } \\
\text { factor } \alpha \text { (TNF- } \alpha \text { ), TNF-RI, TNF-RII, CD14, immunoglobulin } \\
\text { subclasses (IgA, IgM, IgG1-4) at Cytolab }\end{array}$ \\
\hline C-Reactive Protein (CRP) & Serum & Pentameric CRP (pCRP), Monomeric CRP (mCRP) at ApDia \\
\hline Tryptophan and kynurenine pathway intermediates & Serum & $\begin{array}{l}\text { Tryptophan (TRP), kynurenine (KYN), kynurenic acid (KYNA), } \\
\text { 3-hydroxykynurenine (3HK), xanthurenic acid (XAN), } \\
\text { anthranilic acid (AA), quinolinic acid (QUIN), picolinic acid } \\
\text { (PIC), 5-hydroxytryptophan (5-HTRP), 5-hydroxyindoleacetic } \\
\text { acid (5HIAA), nicotinic acid (NAD) at LMU }\end{array}$ \\
\hline Vitamin D & Serum & 25-OH-Vitamin D at LMU \\
\hline $\mathrm{T}$ cells and NK cells & Whole blood & IFN-g, CD4/CD8, CD56/CD3 at ProImmune \\
\hline Genotyping and gene expression & $\begin{array}{l}\text { PAXgene tubes } \\
\text { (RNA), EDTA tubes } \\
\text { (DNA) }\end{array}$ & $\begin{array}{l}\text { Genome-wide genetic factors, genotyping and gene expression at } \\
\text { deCODE Genetics and Biolytix }\end{array}$ \\
\hline Hair cortisol & Hair strains $2-4 \mathrm{~cm}$ & Cortisol measuring chronic stress in hair at TUD \\
\hline
\end{tabular}

See the "Appendix" for laboratory centres

neuropsychiatric disorders, mainly dopamine D2 receptors, in relation to onset or exacerbation of tic disorders.

Genome-wide genetic factors were investigated through the genotyping of participants using peripheral bloodextracted DNA, genotyped on an Illumina Human OmniExpress array, targeting more than 700,000 common variants. Published genome-wide genotyping data for individuals of European descent are used for the control dataset to perform GWAS and identify single gene or gene-gene interactions associated with TS and obsessive-compulsive symptoms. The integration of EMTICS genetics data with existing datasets from parallel efforts from European and American consortia will enable a meta-analysis of a joint dataset with more than 8000 cases with TS and obsessive-compulsive symptoms. In addition, genome-wide gene expression data were investigated using peripheral blood-extracted total RNA, analysed on an Affymetrix Human Transcriptome Array (HTA 2.0), targeting more than 285,000 coding and non-coding and alternatively spliced transcripts to unravel biological pathways that may influence the onset and clinical course of tics activated upon symptom exacerbations and remissions. By combining genotyping and gene expression data with other laboratory measures, we aim to explore the complex interaction between the environmental, immunological and autoimmune factors related to the onset and clinical course of the disorder spectrum. Finally, we determined cortisol levels in hair, which is a biomarker of retrospective chronic stress [78] using a commercially available immunoassay with chemoluminescence detection (CLIA, IBL-Hamburg, Germany) [79, 80].

\section{Sample size calculations}

\section{ONSET study}

The ONSET study originally aimed to recruit 500 participants but managed to include 260 children who are first-degree relatives of patients with a tic disorder, aged 3-10 years. Still this sample size would allow us to obtain an estimated odds ratio of 2.85 for GAS carriers compared to non-carriers with respect to the event "onset", using the pre-study assumptions of an estimated GAS carriage rate during childhood of $15 \%$ [81], and an estimated risk of $30 \%$ [82] for a first-degree relative of a patient with TS or another chronic tic disorder to be affected by a tic disorder or OCD. Assuming a $15 \%$ GAS carriage rate and $30 \%$ risk of TS/OCD in siblings or children of patients with TS at age $3-10$, to obtain an odds ratio of 2.2 for GAS carriers 
compared to non-carriers with respect to the event "onset" with $80 \%$ power and $5 \%$ significance level (two-sided), we calculated a sample size of 319 using the Kelsey method. Assuming a 15\% drop out a sample size of 375 patients allows this number to be achieved.

\section{COURSE study}

The COURSE study includes 715 children and adolescents affected by a tic disorder with an age range of 3-16 years. A target sample size of 700 was based on the following pre-study assumptions: we assumed it would allow us to obtain an estimated odds ratio of 2.45 for GAS carriers compared to non-carriers with respect to the event "exacerbation", assuming an exposure to streptococcal infection of 0.12 and a rate of symptom exacerbation of 0.16 based on a conservative estimate from prior longitudinal studies of TS $[58,59]$. The sample size for the COURSE study was calculated in an identical fashion to the ONSET study.

\section{Genetic and gene expression analyses}

The sample collected in this study will provide $96 \%$ power to detect genetic alleles of frequency $>0.3$ conferring risk $>1.5$, to be analysed using existing tools (e.g. methods implemented in PLINK), as well as by designing novel statistical and data mining techniques, aiming to uncover single gene, gene-gene and gene-environment interaction in TS/OCD pathogenesis. For gene expression group analysis, for a minimum total of 40 samples (20 samples per group), we can reach significant results $\left(p<0.05 / 16,000=3 \times 10^{-6}\right)$ with $98 \%$ power if considering about 16,000 genes remaining after quality control, with the gene(s) showing foldChange $\geq 2$ (up/down regulation).

\section{Statistical analysis}

\section{ONSET study}

We will use logistic regression to evaluate risk factors for developing tics, while survival analysis (Cox proportional hazards model) will be performed to assess the effect of risk factors on the time of developing tics, allowing for clustering of patients within regions. We will also develop a risk model for the risk of new onset of tics in first-degree relatives of patients with chronic tic disorders. Cox proportional hazards regression models (with adjustments for clustering) will be used to develop the risk model. Selection or shrinkage methods may be employed depending on the event rate.

\section{COURSE study}

Data analysis of the COURSE study will be directed at examining the association between the longitudinal course of tics and/or obsessive-compulsive symptoms and various risk factors. Logistic regression will be used to evaluate the effects of risk factors on the risk of tic exacerbation during the same or the subsequent follow-up period, while survival analysis (Cox proportional hazards model) will be performed to assess the effect of risk factors on the time to development of exacerbations, again allowing for clustering of patients within regions.

In both studies, exposure to GAS will be defined as new GAS throat carriage as evaluated on microbiological analysis of the throat specimen ('newly positive' throat swab) OR as significant elevation of anti-streptococcal antibody titres, defined as follows: a significant elevation of anti-streptolysin O titre (ASOT) will be diagnosed when ASO > 200 AND $[\log 10$ (ASOcurrent visit) $-\log 10$ (ASOprior visit) $] \geq 0.2$ (variation between $\log 10$ for two consecutive measurements is higher than or equal to 0.2); a significant elevation of anti-DNAseB will be diagnosed when anti-DNAseB $>300$ AND $[\log 10$ (anti-DNAseBcurrent visit) - $\log 10$ (antiDNAseBprior visit) $] \geq 0.2$ (variation between $\log 10$ for two consecutive measurements is higher than or equal to 0.2 ). In addition to the analyses above, the dataset of the ONSET and COURSE study provides ample opportunities to look into the relationship between immune parameters, infections, psychosocial stress, genetic factors and tic onsets and exacerbations.

\section{Genetic analyses}

Given the diverse origin of the samples that will be included in the produced data sets, particular attention will be paid to statistical techniques that correct for biases that are typically introduced in such analyses, due to population stratification. To assist in this, we will leverage the power of Principal Components Analysis to detect stratification and population substructure. To uncover gene-environment interactions, the case-only design and traditional (e.g. multi-dimensionalityreduction) as well as machine-learning techniques will be used. The resulting list of target susceptibility variants will be refined through gene-network analysis and search of public databases of gene function and ontology. Quantitative trait loci (QTLs) will be identified among the single nucleotide polymorphisms (SNPs) yielding results of high statistical significance to assist in the identification of possible gene pathways that are involved in the susceptibility to the studied disorders. Public gene expression databases (e.g. HapMap data of gene expression from lymphoblastoid cell lines) will be used to that effect. 
We will also mine for gene-gene and gene-environment interactions. In particular, we will attempt to leverage linear discriminant analysis (LDA), a powerful dimensionality reduction technique, to design statistical tests that analyse all the SNP genotypes from a GWAS simultaneously (as opposed to one at the time) and thus identify a subset of SNPs that are most associated or predictive of disease risk. This technique achieves improved performance over single SNP tests.

\section{Gene expression analyses}

Statistical tests (e.g. $T$ tests, ANOVA, ANCOVA) will be performed using Partek, GeneSpring, PAM and BRB-ArrayTools. Special care is going to be taken to correct for potential confounding factors (e.g. ancestry, sex, age). Multiple comparison corrections will be performed using Benjamini and Hochberg False Discovery Rate. A tenfold cross validation will be performed, and the minimum number of genes that predicts a given class will be derived using prediction analysis of microarrays (PAM) which uses a nearest neighbour, smallest shrunken centroid algorithm. Other prediction algorithms including support vector machine methods will be used to confirm these results. Cluster analyses and principal component analyses will be used to visualize how well the genes separate the groups, or to discover new classes.

\section{Data management and data cleaning}

All data were collected on paper and entered into an electronic data base by each site. The data in each site are encrypted and kept in a secured place, and were transmitted in a secure manner, in line with requirements of data protection and data transmission regulations. At each site, a unique code was assigned to recruited members, and tests were delivered to the labs, withholding any identifying information (e.g. name, initials, birth date). After collection of clinical data had been completed we checked all data for improbable values and corrections have been applied where needed by going back to source material. Data access to researchers from outside the EMTICS consortium research shall be granted on fair and reasonable conditions.

\section{Training}

All collaborators were trained in clinical assessments with a focus on the YGTSS by use of video recording of children with tics and in appropriate conduct of research in accordance with ethical requirements. Moreover, throughout the 6.5 years duration of the study we had bi-annual meetings with all investigators in which various aspects of the study were discussed aimed at standardisation of procedures and obtaining agreement. In addition, in case of diagnostic uncertainties, consensus scoring was used within the expert team of the respective clinical centres. The microbiological methods used by different centres to isolate GAS from carriers with low bacterial density were harmonised and improved through a training course and two external quality assessments.

\section{Discussion}

The EMTICS study provides the unique opportunity to analyse data on a broad set of environmental exposures and biological markers related to chronic tic disorders under the umbrella of a common methodological framework. The ONSET cohort of the EMTICS study represents, to the best of our knowledge, the first attempt to capture in a prospective fashion the onset of tics in an at-risk population, i.e. first-degree relatives of probands with a chronic tic disorder. At the same time, the COURSE cohort of the EMTICS study represents, to date, the largest cohort of youth with a chronic tic disorder followed up systematically and in a prospective fashion to capture clinically relevant exacerbations of tics and/or OCD symptoms. It offers therefore the possibility to investigate, with larger power than previously sought, the potential determinants of severity fluctuations within these disorders. This type of design and dataset offers an as yet unforeseen opportunity to develop prediction models and algorithms that quantify the risk of onset or exacerbation of tic disorders based on environmental exposures and gene-environment interactions. Such predictive algorithms would then need further validation in other populations before being introduced in routine clinical activity.

The previous studies that have tackled the multifactorial influence of environment and gene-environment interactions upon the natural history of chronic tic disorders were either population-based studies that used national health- or health insurance claims-based registries, or single- or multicentre, clinic-based, prospective studies. Population-based studies have reported associations of TS/chronic tic disorders and common comorbidities with a very specific environmental exposure such as GAS infections [50-54], with little or no insight on interactions between exposures. Although the majority of these studies reported an increased risk of tic disorders for individuals with a history of previous streptococcal throat infection ranging between 1.2 and 2.2, these estimates vary across studies, and in at least one study [54] the association was not confirmed. The variability across health data registry studies in the definition of GAS infections as well as in the source and methodology of data collection from different care providers possibly reasons for the discrepancies across risk estimates. Our prospective, hypothesis-based study design overcomes this limitation, using a strict and standardized, homogenous and quality-controlled 
determination of GAS colonisation. Moreover, our study design guarantees homogeneity, compared to populationbased studies, also with respect to the assessment of tic disorders and concurrent symptoms through a trained group of clinicians, minimising medical surveillance bias and recall bias. We acknowledge, however, that some of the data collected in EMTICS, e.g. those on pre- and perinatal adversities might also suffer from a certain degree of recall bias, in the lack of supporting medical documentation. Another advantage with respect to previous population-based studies is the possibility for correlating clinically relevant events or exposures to biological measures, which is key to understanding multifactorial interactions and elaborate mechanistic explanations for the observed associations. The main limitation compared to population-based studies is the risk of a larger type II error, particularly in the ONSET cohort, linked to smaller study power.

Previous prospective, clinic-based studies enrolled smaller $(N<50$ with the exception of [54] patient cohorts [55-59], often aiming to isolate a subgroup of patients with PANDAS from a clinic-based population of patients with a 'garden variety' tic disorders. The research questions of the EMTICS study do not refer to the PANDAS or the PANS spectrum, and indeed clinical data gathered in EMTICS are insufficient to diagnose PANS accurately. Compared to previous clinic-based studies, EMTICS relies on rating scale based data to confirm or exclude exacerbations of tics, thus minimising a potential bias resulting from subjective definitions of exacerbation based on the judgement of individual clinicians. Previous clinic-based studies have, in some cases [56, 59], applied a more intensive clinical follow-up over a period of 24 months, although this applied to a population of less than 90 patients. Logistic limitations related to the international composition of the EMTICS list of enrolment sites did not allow such an intensive follow-up for a very large population. We partially mitigated this potential limitation in the COURSE cohort by replacing highly frequent clinic visits with the use of structured weekly diaries and bi-monthly telephone interviews in between clinic visits. A caveat of this logistic limitation is the greater reliance on self-reported data. Finally, prospective clinic-based studies did not analyse in detail interactions between exposures, with the only exception of the study by Lin et al. [59], which evaluated the interaction between GAS infections and psychosocial stress levels on a relatively small population of 45 cases with TS and/or OCD and 41 matched control subjects. The design of EMTICS appears highly promising to test this interaction with a substantially greater statistical power.

Albeit comprehensive, the EMTICS study does not include all the potential pathophysiological biomarkers of TS that could be influenced by environmental exposures and gene-environment interactions, e.g. imaging of brain networks or microbiome data. The EMTICS research platform has, however, included parallel studies of animal models that aim to replicate some of the pathophysiological constructs explored by clinical studies. Some of these animal model data have already been published [83-85] and a detailed explanation of these pre-clinical studies is beyond the scope of this article. We acknowledge that other comorbid behavioural symptoms, e.g. ADHD symptoms, may also fluctuate over time in patients with tic disorders; however, we focused on the prospective evaluation of tics and obsessive-compulsive symptoms, as preliminary studies reported a similar short-term influence of putative environmental risk factors (psychosocial stress, GAS infections) on both tic and obsessive-compulsive symptom severity.

Lastly, one concern regarding the gene expression study in EMTICS has been whether peripheral blood can mirror the neuronal tissue, when it comes to detecting altered gene expression profiles that could be relevant to brain pathology. However, recent data support the fact that changes of gene expression in blood may actually constitute distinct molecular signatures of not only clearly hereditary disorders (e.g. neurofibromatosis type I, tuberous sclerosis complex 2, Down's syndrome [86]; Huntington's disease Borovecki [87]), but also complex disorders of the brain (multiple sclerosis [88]; migraine and TS [89, 90]). Thus, there has been considerable effort in determining whether blood gene expression profiling can provide surrogate markers for neurological diseases, including TS, especially when considering (1) the inaccessibility of the actual diseased tissue, and (2) the often very young age of cases with TS participating in research studies, such as EMTICS. In fact, it was recently shown that distinct gene expression changes were correlated with tic severity in medicated versus unmedicated TS cases [91], whereas specific molecular signatures were correlated with inattention and hyperactivity/impulsivity, a very common TS comorbidity, in individuals with TS [92]. Therefore, we believe that EMTICS may further elucidate the genetic background underlying tic disorders and to may shed light on how these interact with environmental factors influencing the onset and clinical course of tic disorders.

We conclude that this study, the first European study of TS of this size granted by the EU, provides innovative and unique avenues to address the aetiology of chronic tic disorders, and we hope that it will represent the consolidation of a stable collaborative research and clinical environment on TS and related disorders in Europe.

Acknowledgements The authors are deeply grateful to all children and their parents who willingly participated to make this research possible. This project has received funding from the European Union's Seventh Framework Programme for research, technological development and demonstration under Grant agreement no. 278367. Schrag was supported by the National Institute for Health Research UCLH Biomedical Research Centre, and Müller, Burger, Schnell and Weidinger by Stiftung Immunität und Seele. This research was supported by the 
National Institute for Health Research Biomedical Research Centre at Great Ormond Street Hospital for Children NHS Foundation Trust and University College London (Heyman); partially sponsored by GSK Vaccines (Margarit, Bartolini); and Deutsche Forschungsgemeinschaft (DFG): projects 1692/3-1, 4-1 (Münchau). We thank all members of the EMTICS collaborative group for their continued commitment to this project and in particular all colleagues at the various study centres who contributed to data collection and/or management: Julie E Bruun, Judy Grejsen, Christine L Ommundsen, Mette Rubæk (Capital Region Psychiatry, Copenhagen, Denmark); Stephanie Enghardt (TUD Dresden, Germany); Stefanie Bokemeyer, Christiane Driedger-Garbe, Cornelia Reichert (MHH Hannover, Germany); Thomas Duffield (LMU München, Germany); Jennifer Tübing, Jenny Schmalfeld (Lübeck University, Germany); Franciska Gergye, Margit Kovacs, Reka Vidomusz (Vadaskert Budapest, Hungary); Miri Carmel, Silvana Fennig, Ella Gev, Nathan Keller, Elena Michaelovsky, Matan Nahon, Chen Regev, Tomer Simcha, Gill Smollan, Avi Weizman (Tel Aviv, PetahTikva, Israel); Giuseppe Gagliardi (Bari, Italy); and Marco Pataracchia, Simona Recchia, Giovanna Alfarone (ISS Rome, Italy); Mascha van den Akker, Marieke Messchendorp, Florianne Rademaker, Anne Marie Stolte (UMCG Groningen, Netherlands); Maria Teresa Cáceres, Fátima Carrillo, Pilar Gómez-Garre, Ángela Periañez Vasco, Laura Vargas (Seville, Spain); and all who may not have been mentioned. We also express our gratitude to the advisory board Danielle Cath, James Leckman, and Angela Vincent; as well as Mary Robertson; and the laboratories of Raf Berghmans (ApDia), Adrian Urwyler (Cytolab) and Andrew Waller and Nikolai Schwabe (ProImmune) for their support.

\section{Compliance with ethical standards}

Conflict of interest Bartolini and Margarit report that they were employee of Novartis Vaccines at the start of the study. Following the acquisition of Novartis Vaccines by the GSK group of companies in March, 2015, Bartolini and Margarit are now employees of the GSK group of companies. Müller-Vahl received funding for research from the EU (FP7-PEOPLE-2012-ITN No. 316978), the German Research Society (DFG: GZ MU 1527/3-1), the German Ministry of Education and Research (BMBF: 01KG1421), the National Institute of Mental Health (NIMH), GW, Almirall, Abide Therapeutics, and Therapix Biosiences, and consultant's honoraria from Abide Therapeutics, Fundacion Canna, and Therapix Biosiences. Walitza has received lecture honoraria from Eli-Lilly, Opopharma in the last 5 years. On behalf of all other authors, the corresponding author declares that the other authors have no conflict of interest.

Ethical standards This study was approved by the institutional review boards at all participating clinical centres in accordance with the ethical standards laid down in the 1964 Declaration of Helsinki and its later amendments. All parents (or the legal guardian) provided written informed consent and the participating child written or oral assent before entering the study.

Open Access This article is distributed under the terms of the Creative Commons Attribution 4.0 International License (http://creativeco mmons.org/licenses/by/4.0/), which permits unrestricted use, distribution, and reproduction in any medium, provided you give appropriate credit to the original author(s) and the source, provide a link to the Creative Commons license, and indicate if changes were made.

\section{Appendix}

The EMTICS group members are Zacharias Anastasiou ${ }^{1}$, Alan Apter ${ }^{2}$, Valentina Baglioni ${ }^{3}$, Juliane Ball ${ }^{4}$, Erika Bartolini $^{5}$, Noa Benaroya-Milshtein ${ }^{2}$, Benjamin Bodmer ${ }^{6}$, Emese Bognar ${ }^{7}$, Bianka Burger ${ }^{8,9}$, Judith Buse ${ }^{6}$, Maura Buttiglione $^{10}$, Francesco Cardona ${ }^{3}$, Marta Correa Vela ${ }^{11}$, Roberta Creti ${ }^{12}$, Andrea Dietrich ${ }^{13}$, Nanette M. Debes ${ }^{14}$, Androulla Efstratiou ${ }^{15}$, Maria Cristina Ferro ${ }^{16}$, Carolin Fremer ${ }^{17}$, Blanca Garcia-Delgar ${ }^{18}$, Maria Gariup ${ }^{19,20}$, Marianthi Georgitsi ${ }^{21,22}$, Mariangela Gulisano ${ }^{16}$, Annelieke Hagen $^{23,24}$, Julie Hagstrøm ${ }^{25}$, Tammy J. Hedderly ${ }^{26}$, Isobel Heyman $^{27}$, Pieter J. Hoekstra ${ }^{13}$, Chaim Huyser ${ }^{23,24}$, Monica Imperi $^{12}$, Iordanis Karagiannidis ${ }^{21}$, Giovanni Laviola ${ }^{28}$, Simone Macri $^{28}$, Marcos Madruga-Garrido ${ }^{29}$, Immaculada Margarit ${ }^{5}$, Anna Marotta ${ }^{30}$, Davide Martino ${ }^{31}$, Ute C. Meier $^{32}$, Pablo Mir ${ }^{11}$, Natalie Moll ${ }^{33}$, Astrid Morer ${ }^{18,34,35}$, Norbert Müller ${ }^{8,9}$, Kirsten Müller-Vahl ${ }^{17}$, Alexander Münchau $^{36}$, Peter Nagy ${ }^{7}$, Valeria Neri ${ }^{3}$, Thaïra J.C. Openneer ${ }^{13}$, Graziella Orefici ${ }^{37}$, Peristera Paschou ${ }^{38}$, Alessandra Pellico ${ }^{16}$, Onofrio Petruzzelli ${ }^{10}$, Kerstin J. Plessen ${ }^{25,39}$, Cesare Porcelli ${ }^{30}$, Marina Redondo ${ }^{18}$, Renata Rizzo ${ }^{16}$, Paolo Roazzi $^{40}$, Veit Roessner ${ }^{6}$, Daphna Ruhrman ${ }^{2}$, Jaana M.L. Schnell $^{8}$, Anette Schrag ${ }^{41}$, Gregor A. Schütze ${ }^{33}$, Markus J. Schwarz $^{33}$, Paola Rosaria Silvestri ${ }^{3}$, Liselotte Skov $^{42}$, Tamar Steinberg ${ }^{2}$, Sara Stöber ${ }^{42}$, Friederike Tagwerker Gloor ${ }^{4}$, Marco Tallon ${ }^{43}$, Zsanett Tarnok ${ }^{7}$, Victoria L. Turner ${ }^{26}$, Susanne Walitza ${ }^{4}$, Elif Weidinger ${ }^{8}$, and Martin L. Woods ${ }^{26}$

${ }^{1}$ Department of Statistical Science, University College London, London, UK

${ }^{2}$ Child and Adolescent Psychiatry Department, Schneider Children's Medical Center of Israel, affiliated to Sackler Faculty of Medicine, Tel Aviv University, Petah-Tikva, Israel

${ }^{3}$ University La Sapienza of Rome, Department of Human Neurosciences, Rome, Italy

${ }^{4}$ Clinic of Child and Adolescent Psychiatry and Psychotherapy, University of Zurich, Zurich, Switzerland

${ }^{5} \mathrm{GSK}$, Siena, Italy

${ }^{6}$ Department of Child and Adolescent Psychiatry, Faculty of Medicine of the TU Dresden, Dresden, Germany

${ }^{7}$ Vadaskert Child and Adolescent Psychiatric Hospital, Budapest, Hungary

${ }^{8}$ Department of Psychiatry and Psychotherapy, University Hospital, LMU Munich, Munich, Germany

${ }^{9}$ Marion von Tessin Memory-Zentrum gGmbH, Munich, Germany

${ }^{10}$ University of Bari "Aldo Moro", Medical School, Department of Biological Sciences and Human Oncology, Bari, Italy

${ }^{11}$ Unidad de Trastornos del Movimiento, Servicio de Neurología y Neurofisiología Clinica. Instituto de Biomedicina 
de Sevilla (IBiS), Hospital Universitario Virgen del Rocio/ CSIC/Universidad de Sevilla, Seville, Spain

${ }^{12}$ Department of Infectious Diseases, Istituto Superiore di Sanità, Rome, Italy

${ }^{13}$ University of Groningen, University Medical Center Groningen, Department of Child and Adolescent Psychiatry, Groningen, the Netherlands

${ }^{14}$ Paediatric Department, Herlev University Hospital, Herlev, Denmark

${ }^{15}$ WHO Global Collaborating Centre for Reference and Research on Diphtheria and Streptococcal Infections; Reference Microbiology, Directorate National Infection Service, Public Health England, London, UK

${ }^{16}$ Child Neuropsychiatry Section, Department of Clinical and Experimental Medicine, School of Medicine, Catania University, Catania, Italy

${ }^{17}$ Clinic of Psychiatry, Socialpsychiatry and Psychotherapy, Hannover Medical School, Hannover, Germany

${ }^{18}$ Department of Child and Adolescent Psychiatry and Psychology, Institute of Neurosciences, Hospital Clinic Universitari, Barcelona, Spain

${ }^{19}$ University of Barcelona, Barcelona, Spain

${ }^{20}$ Copenhagen Psychiatric Center, Intensive Inpatient Unit, Copenhagen, Denmark

${ }^{21}$ Department of Molecular Biology and Genetics, Democritus University of Thrace, Alexandroupoli, Greece

${ }^{22}$ Department of Medicine, Aristotle University of

Thessaloniki, Thessaloniki, Greece

${ }^{23}$ De Bascule, Academic Center for Child and Adolescent Psychiatry, Amsterdam, the Netherlands

${ }^{24}$ Academic Medical Center, Department of Child and Adolescent Psychiatry, Amsterdam, the Netherlands

${ }^{25}$ Child and Adolescent Mental Health Center, Mental Health Services, Capital Region of Denmark and University of Copenhagen, Copenhagen, Denmark

${ }^{26}$ Evelina London Children's Hospital GSTT, Kings

Health Partners AHSC, London, UK

${ }^{27}$ Great Ormond Street Hospital for Children, and UCL Institute of Child Health, London, UK

${ }^{28}$ Reference Centre for Behavioural Sciences and Mental Health, Istituto Superiore di Sanità, Roma, Italy

${ }^{29}$ Sección de Neuropediatría, Instituto de Biomedicina de Sevilla (IBiS), Hospital Universitario Virgen del Rocío/ CSIC/Universidad de Sevilla, Seville, Spain

${ }^{30}$ Azienda Sanitaria Locale di Bari, Mental Health Department, Child and Adolescent Service of Bari Metropolitan Area, Bari, Italy

${ }^{31}$ Department of Clinical Neurosciences, University of Calgary, Calgary, Canada

${ }^{32}$ Blizard Institute, Queen Mary University of London, London, UK

${ }^{33}$ Institute of Laboratory Medicine, University Hospital, LMU Munich, Munich, Germany
${ }^{34}$ Institut d'Investigacions Biomediques August Pi i Sunyer (IDIBAPS), Barcelona, Spain

${ }^{35}$ Centro de Investigacion en Red de Salud Mental (CIBERSAM), Instituto Carlos III, Spain

${ }^{36}$ Institute of Neurogenetics, University of Lübeck, Lübeck, Germany

${ }^{37}$ formerly Department of Infectious Diseases, Istituto Superiore di Sanità, Rome, Italy

${ }^{38}$ Department of Biological Sciences, Purdue University, West Lafayette, USA

${ }^{39}$ Service of Child and Adolescent Psychiatry, Department of Psychiatry, University Medical Center, University of Lausanne, Lausanne, Switzerland

${ }^{40}$ Health Technology Assessment Centre, Istituto Superiore di Sanità, Rome, Italy

${ }^{41}$ Department of Clinical Neuroscience, UCL Institute of Neurology, University College London, London, UK

${ }^{42}$ Concentris research management $\mathrm{GmbH}$, Fürstenfeldbruck, Germany

${ }^{43}$ IT Service, Istituto Superiore di Sanità, Rome, Italy

Laboratory centres and research management:

${ }^{1}$ Advanced Practical Diagnostics (ApDia), Belgium

(Raf Berghmans)

${ }^{2}$ deCODE Genetics, Reykjavik, Iceland

${ }^{3}$ Biolytics, Witterswil, Switzerland

${ }^{4}$ concentris research management $\mathrm{GmbH}$, Germany (Ameli Schwalber, Sara Stöber)

${ }^{5}$ Cytolab, Switzerland (Adrian Urwyler)

${ }^{6}$ GSK Vaccines, Siena, Italy (Immaculada Margarit, Erika Bartolini)

${ }^{7}$ Health Technology Assessment Centre, National Institute of Health (Istituto Superiore di Sanità, ISS), Italy (Paolo Roazzi)

${ }^{8}$ LMU Munich, Germany (Markus Schwarz)

${ }^{9}$ University of Groningen, University Medical Center Groningen, Netherlands (coordinating site, Pieter J. Hoekstra)

${ }^{10}$ ProImmune, UK (Andrew Waller, Nikolai Schwabe)

${ }^{11}$ TU Dresden, Germany (Veit Roessner)

${ }^{12}$ University of Bari "Aldo Moro", Italy (Maura Buttiglione)

\section{References}

1. Arlington VA (2013) American Psychiatric Association. Diagnostic and statistical manual of mental disorders, 5th edn. American Psychiatric Publication, Washington, DC

2. Knight T, Steeves T, Day L, Lowerison M, Jette N, Pringsheim $\mathrm{T}$ (2012) Prevalence of tic disorders: a systematic review and meta-analysis. Pediatr Neurol 47(2):77-90

3. Robertson MM (2008) The prevalence and epidemiology of Gilles de la Tourette syndrome. Part 2: tentative explanations 
for differing prevalence figures in GTS, including the possible effects of psychopathology, aetiology, cultural differences, and differing phenotypes. J Psychosom Res 65(5):473-486

4. Robertson MM (2015) A personal 35 year perspective on Gilles de la Tourette syndrome: assessment, investigations, and management. Lancet Psychiatry 2(1):88-104

5. Altman G, Staley JD, Wener P (2009) Children with Tourette disorder: a follow-up study in adulthood. J Nerv Ment Dis 197(5):305-310

6. Eddy CM, Rizzo R, Gulisano M, Agodi A, Barchitta M, Cali $P$ et al (2011) Quality of life in young people with Tourette syndrome: a controlled study. J Neurol 258(2):291-301

7. Cavanna AE, David K, Bandera V, Termine C, Balottin U, Schrag A et al (2013) Health-related quality of life in Gilles de la Tourette syndrome: a decade of research. Behav Neurol 27(1):83-93

8. Jalenques I, Galland F, Malet L, Morand D, Legrand G, Auclair C et al (2012) Quality of life in adults with Gilles de la Tourette Syndrome. BMC Psychiatry 12:109

9. Jalenques I, Auclair C, Morand D, Legrand G, Marcheix M, Ramanoel C et al (2017) Health-related quality of life, anxiety and depression in parents of adolescents with Gilles de la Tourette syndrome: a controlled study. Eur Child Adolesc Psychiatry 26(5):603-617

10. Cheung MY, Shahed J, Jankovic J (2007) Malignant Tourette syndrome. Mov Disord 22(12):1743-1750

11. Mataix-Cols D, Isomura K, Perez-Vigil A, Chang Z, Ruck C, Larsson KJ et al (2015) Familial risks of Tourette syndrome and chronic tic disorders. A population-based cohort study. JAMA Psychiatry 72(8):787-793

12. Zilhao NR, Olthof MC, Smit DJ, Cath DC, Ligthart L, Mathews CA et al (2017) Heritability of tic disorders: a twin-family study. Psychol Med 47(6):1085-1096

13. Scharf JM, Yu D, Mathews CA, Neale BM, Stewart SE, Fagerness JA et al (2013) Genome-wide association study of Tourette's syndrome. Mol Psychiatry 18(6):721-728

14. Paschou P, Yu D, Gerber G, Evans P, Tsetsos F, Davis LK et al (2014) Genetic association signal near NTN4 in Tourette syndrome. Ann Neurol 76(2):305-310

15. Qi Y, Zheng Y, Li Z, Xiong L (2017) Progress in Genetic Studies of Tourette's Syndrome. Brain Sci 7(10):E134

16. Huang AY, Yu D, Davis LK, Sul JH, Tsetsos F, Ramensky V et al (2017) Rare copy number variants in NRXN1 and CNTN6 increase risk for Tourette syndrome. Neuron 94(6):1101-1111

17. Eriguchi Y, Kuwabara H, Inai A, Kawakubo Y, Nishimura F, Kakiuchi $\mathrm{C}$ et al (2017) Identification of candidate genes involved in the etiology of sporadic Tourette syndrome by exome sequencing. Am J Med Genet B Neuropsychiatr Genet 174(7):712-723

18. Sun N, Nasello C, Deng L, Wang N, Zhang Y, Xu Z et al (2017) The PNKD gene is associated with Tourette disorder or Tic disorder in a multiplex family. Mol Psychiatry. https://doi.org/10.1038/ MP.2017.179

19. Sundaram SK, Huq AM, Sun Z, Yu W, Bennett L, Wilson BJ et al (2011) Exome sequencing of a pedigree with Tourette syndrome or chronic tic disorder. Ann Neurol 69(5):901-904

20. Willsey AJ, Fernandez TV, Yu D, King RA, Dietrich A, Xing J et al (2017) De novo coding variants are strongly associated with Tourette disorder. Neuron 94(3):486-499

21. Lit L, Enstrom A, Sharp FR, Gilbert DL (2009) Age-related gene expression in Tourette syndrome. J Psychiatr Res 43(3):319-330

22. Tian Y, Liao IH, Zhan X, Gunther JR, Ander BP, Liu D et al (2011) Exon expression and alternatively spliced genes in Tourette Syndrome. Am J Med Genet B Neuropsychiatr Genet 156B(1):72-78
23. Tian Y, Gunther JR, Liao IH, Liu D, Ander BP, Stamova BS et al (2011) GABA- and acetylcholine-related gene expression in blood correlate with tic severity and microarray evidence for alternative splicing in Tourette syndrome: a pilot study. Brain Res 24(1381):228-236

24. Muller-Vahl KR, Loeber G, Kotsiari A, Muller-Engling L, Frieling $H$ (2017) Gilles de la Tourette syndrome is associated with hypermethylation of the dopamine D2 receptor gene. J Psychiatr Res 86:1-8

25. Leivonen S, Voutilainen A, Chudal R, Suominen A, Gissler M, Sourander A (2016) Obstetric and neonatal adversities, parity, and Tourette syndrome: a nationwide registry. J Pediatr 171:213-219

26. Browne HA, Modabbernia A, Buxbaum JD, Hansen SN, Schendel DE, Parner ET et al (2016) Prenatal maternal smoking and increased risk for Tourette syndrome and chronic tic disorders. $\mathbf{J}$ Am Acad Child Adolesc Psychiatry 55(9):784-791

27. Chao TK, Hu J, Pringsheim T (2014) Prenatal risk factors for Tourette syndrome: a systematic review. BMC Pregnancy Childbirth 30(14):53

28. Abdulkadir M, Tischfield JA, King RA, Fernandez TV, Brown LW, Cheon KA et al (2016) Pre- and perinatal complications in relation to Tourette syndrome and co-occurring obsessive-compulsive disorder and attention-deficit/hyperactivity disorder. J Psychiatr Res 82:126-135

29. Brander G, Rydell M, Kuja-Halkola R, Fernández de la Cruz L, Lichtenstein P, Serlachius E, Rück C, Almqvist C, D’Onofrio BM, Larsson H, Mataix-Cols D (2018) Perinatal risk factors in Tourette's and chronic tic disorders: a total population sibling comparison study. Mol Psychiatry 23(5):1189-1197

30. Buse J, Kirschbaum C, Leckman JF, Münchau A, Roessner V (2014) The Modulating role of stress in the onset and course of Tourette's syndrome: a review. Behav Modif 38(2):184-216

31. Lin H, Katsovich L, Ghebremichael M, Findley DB, Grantz H, Lombroso PJ et al (2007) Psychosocial stress predicts future symptom severities in children and adolescents with Tourette syndrome and/or obsessive-compulsive disorder. J Child Psychol Psychiatry 48(2):157-166

32. Corbett BA, Mendoza SP, Baym CL, Bunge SA, Levine S (2008) Examining cortisol rhythmicity and responsivity to stress in children with Tourette syndrome. Psychoneuroendocrinology 33(6):810-820

33. Buse J, Enghardt S, Kirschbaum C, Ehrlich S, Roessner V (2016) Tic frequency decreases during short-term psychosocial stress - an experimental study on children with tic disorders. Front Psychiatry 17(7):84

34. Dalsgaard S, Waltoft BL, Leckman JF, Mortensen PB (2015) Maternal history of autoimmune disease and later development of tourette syndrome in offspring. J Am Acad Child Adolesc Psychiatry 54(6):495-501

35. Mataix-Cols D, Frans E, Perez-Vigil A, Kuja-Halkola R, Gromark C, Isomura K et al (2017) A total-population multigenerational family clustering study of autoimmune diseases in obsessive-compulsive disorder and Tourette's/chronic tic disorders. Mol Psychiatry. https://doi.org/10.1038/mp.2017.215

36. Martino D, Zis P, Buttiglione M (2015) The role of immune mechanisms in Tourette syndrome. Brain Res 18(1617):126-143

37. Perez-Vigil A, Fernandez dlC, Brander G, Isomura K, Gromark C, Mataix-Cols D (2016) The link between autoimmune diseases and obsessive-compulsive and tic disorders: a systematic review. Neurosci Biobehav Rev 71:542-562

38. Lennington JB, Coppola G, Kataoka-Sasaki Y, Fernandez TV, Palejev D, Li Y et al (2016) Transcriptome analysis of the human striatum in Tourette syndrome. Biol Psychiatry 79(5):372-382

39. Kumar A, Williams MT, Chugani HT (2015) Evaluation of basal ganglia and thalamic inflammation in children with pediatric 
autoimmune neuropsychiatric disorders associated with streptococcal infection and tourette syndrome: a positron emission tomographic (PET) study using 11C-[R]-PK11195. J Child Neurol 30(6):749-756

40. Frick L, Pittenger C (2016) Microglial dysregulation in OCD, Tourette Syndrome, and PANDAS. J Immunol Res 2016:8606057. https://doi.org/10.1155/2016/8606057

41. Swedo SE, Leonard HL, Garvey M, Mittleman B, Allen AJ, Perlmutter $S$ et al (1998) Pediatric autoimmune neuropsychiatric disorders associated with streptococcal infections: clinical description of the first 50 cases. Am J Psychiatry 155(2):264-271

42. Swedo SE, Seidlitz J, Kovacevic M, Latimer ME, Hommer R, Lougee L et al (2015) Clinical presentation of pediatric autoimmune neuropsychiatric disorders associated with streptococcal infections in research and community settings. J Child Adolesc Psychopharmacol 25(1):26-30

43. Murphy TK, Patel PD, McGuire JF, Kennel A, Mutch PJ, ParkerAthill EC et al (2015) Characterization of the pediatric acuteonset neuropsychiatric syndrome phenotype. J Child Adolesc Psychopharmacol 25(1):14-25

44. Sigra S, Hesselmark E, Bejerot S (2018) Treatment of PANDAS and PANS: a systematic review. Neurosci Biobehav Rev 86:51-65

45. Cox CJ, Sharma M, Leckman JF, Zuccolo J, Zuccolo A, Kovoor A et al (2013) Brain human monoclonal autoantibody from sydenham chorea targets dopaminergic neurons in transgenic mice and signals dopamine D2 receptor: implications in human disease. $\mathrm{J}$ Immunol 191(11):5524-5541

46. Singer HS, Mascaro-Blanco A, Alvarez K, Morris-Berry C, Kawikova I, Ben-Pazi H et al (2015) Neuronal antibody biomarkers for Sydenham's chorea identify a new group of children with chronic recurrent episodic acute exacerbations of tic and obsessive compulsive symptoms following a streptococcal infection. PLoS One 10(3): e0120499

47. Hesselmark E, Bejerot S (2017) Biomarkers for diagnosis of pediatric acute neuropsychiatric syndrome (PANS)—-sensitivity and specificity of the Cunningham Panel. J Neuroimmunol 15(312):31-37

48. Stagi S, Lepri G, Rigante D, Matucci CM, Falcini F (2017) Crosssectional evaluation of plasma vitamin d levels in a large cohort of italian patients with pediatric autoimmune neuropsychiatric disorders associated with streptococcal infections. J Child Adolesc Psychopharmacol. https://doi.org/10.1089/cap.2016.0159

49. Dale RC, Merheb V, Pillai S, Wang D, Cantrill L, Murphy TK et al (2012) Antibodies to surface dopamine-2 receptor in autoimmune movement and psychiatric disorders. Brain 135(Pt 11):3453-3468

50. Mell LK, Davis RL, Owens D (2005) Association between streptococcal infection and obsessive-compulsive disorder, Tourette's syndrome, and tic disorder. Pediatrics 116(1):56-60

51. Leslie DL, Kozma L, Martin A, Landeros A, Katsovich L, King RA et al (2008) Neuropsychiatric disorders associated with streptococcal infection: a case-control study among privately insured children. J Am Acad Child Adolesc Psychiatry 47(10):1166-1672

52. Wang HC, Lau CI, Lin CC, Chang A, Kao CH (2016) Group A streptococcal infections are associated with increased risk of pediatric neuropsychiatric disorders: a Taiwanese population-based cohort study. J Clin Psychiatry 77(7):e848-e854

53. Orlovska S, Vestergaard $\mathrm{CH}$, Bech $\mathrm{BH}$, Nordentoft M, Vestergaard M, Benros ME (2017) Association of streptococcal throat infection with mental disorders: testing key aspects of the PANDAS hypothesis in a nationwide study. JAMA Psychiatry 74(7):740-746

54. Schrag A, Gilbert R, Giovannoni G, Robertson MM, Metcalfe C, Ben-Shlomo Y (2009) Streptococcal infection, Tourette syndrome, and OCD: is there a connection? Neurology 73(16):1256-1263
55. Martino D, Chiarotti F, Buttiglione M, Cardona F, Creti R, Nardocci $\mathrm{N}$ et al (2011) The relationship between group A streptococcal infections and Tourette syndrome: a study on a large servicebased cohort. Dev Med Child Neurol 53(10):951-957

56. Kurlan R, Johnson D, Kaplan EL (2008) Streptococcal infection and exacerbations of childhood tics and obsessive-compulsive symptoms: a prospective blinded cohort study. Pediatrics 121(6):1188-1197

57. Murphy TK, Sajid M, Soto O, Shapira N, Edge P, Yang M et al (2004) Detecting pediatric autoimmune neuropsychiatric disorders associated with streptococcus in children with obsessivecompulsive disorder and tics. Biol Psychiatry 55(1):61-68

58. Luo F, Leckman JF, Katsovich L, Findley D, Grantz H, Tucker DM et al (2004) Prospective longitudinal study of children with tic disorders and/or obsessive-compulsive disorder: relationship of symptom exacerbations to newly acquired streptococcal infections. Pediatrics 113(6):e578-e585

59. Leckman JF, King RA, Gilbert DL, Coffey BJ, Singer HS, Dure LS et al (2011) Streptococcal upper respiratory tract infections and exacerbations of tic and obsessive-compulsive symptoms: a prospective longitudinal study. J Am Acad Child Adolesc Psychiatry $50(2): 108-118$

60. Lin H, Williams KA, Katsovich L, Findley DB, Grantz H, Lombroso PJ et al (2010) Streptococcal upper respiratory tract infections and psychosocial stress predict future tic and obsessive-compulsive symptom severity in children and adolescents with Tourette syndrome and obsessive-compulsive disorder. Biol Psychiatry 67(7):684-691

61. Bombaci M, Grifantini R, Mora M, Reguzzi V, Petracca R, Meoni E et al (2009) Protein array profiling of tic patient sera reveals a broad range and enhanced immune response against Group A Streptococcus antigens. PLoS One 4(7):e6332

62. American Psychiatric Association (2000) American Psychiatric Association. Diagnostic and statistical manual of mental disorders, 4th edn. DSM IV-TR, Washington, DC

63. Peterson BS (1996) Considerations of natural history and pathophysiology in the psychopharmacology of Tourette's syndrome. J Clin Psychiatry 57(Suppl 9):24-34

64. Leckman JF, Riddle MA, Hardin MT, Ort SI, Swartz KL, Stevenson J et al (1989) The Yale Global Tic Severity Scale: initial testing of a clinician-rated scale of tic severity. J Am Acad Child Adolesc Psychiatry 28(4):566-573

65. Goodman WK, Price LH, Rasmussen SA, Mazure C, Delgado P, Heninger GR et al (1989) The yale-brown obsessive compulsive scale. II. Validity. Arch Gen Psychiatry 46(11):1012-1016

66. Clinical Global Impression (CGI) (1976) ECDEU assessment manual for psychopharmacology. In: Guy W (ed) U.S. Department of Health Education and Welfare, Rockville, MD

67. Dietrich A, Fernandez TV, King RA, State MW, Tischfield JA, Hoekstra PJ et al (2015) The Tourette International Collaborative Genetics (TIC Genetics) study, finding the genes causing Tourette syndrome: objectives and methods. Eur Child Adolesc Psychiatry 24(2):141-151

68. Walkup JT, Leckman JF, Price RA, Hardin M, Ort SI, Cohen DJ (1988) The relationship between obsessive-compulsive disorder and Tourette's syndrome: a twin study. Psychopharmacol Bull 24(3):375-379

69. Cohen S, Kamarck T, Mermelstein R (1983) A global measure of perceived stress. J Health Soc Behav 24(4):385-396

70. Goodman R (1999) The extended version of the Strengths and Difficulties Questionnaire as a guide to child psychiatric caseness and consequent burden. J Child Psychol Psychiatry 40(5):791-799

71. Goodman R (2001) Psychometric properties of the strengths and difficulties questionnaire. J Am Acad Child Adolesc Psychiatry 40(11):1337-1345 
72. Gau SS, Shang CY, Liu SK, Lin CH, Swanson JM, Liu YC et al (2008) Psychometric properties of the Chinese version of the Swanson, Nolan, and Pelham, version IV scale-parent form. Int J Methods Psychiatr Res 17(1):35-44

73. Swanson JM, Kraemer HC, Hinshaw SP, Arnold LE, Conners CK, Abikoff HB et al (2001) Clinical relevance of the primary findings of the MTA: success rates based on severity of ADHD and ODD symptoms at the end of treatment. J Am Acad Child Adolesc Psychiatry 40(2):168-179

74. Ehlers S, Gillberg C, Wing L (1999) A screening questionnaire for Asperger syndrome and other high-functioning autism spectrum disorders in school age children. J Autism Dev Disord 29(2):129-141

75. Ravens-Sieberer U, Bullinger M (1998) Assessing health-related quality of life in chronically ill children with the German KINDL: first psychometric and content analytical results. Qual Life Res 7(5):399-407

76. Woods DW, Piacentini J, Himle MB, Chang S (2005) Premonitory Urge for Tics Scale (PUTS): initial psychometric results and examination of the premonitory urge phenomenon in youths with Tic disorders. J Dev Behav Pediatr 226(6):397-403

77. Cardona F, Orefici G (2001) Group A streptococcal infections and tic disorders in an Italian pediatric population. J Pediatr 138(1):71-75

78. Stalder T, Kirschbaum C (2012) Analysis of cortisol in hairstate of the art and future directions. Brain Behav Immun 26(7):1019-1129

79. Groeneveld MG, Vermeer HJ, Linting M, Noppe G, van Rossum EF, van IJzendoorn MH (2013) Children's hair cortisol as a biomarker of stress at school entry. Stress 16(6):711-715

80. Gray NA, Dhana A, Van Vyver LD, Van Wyk J, Khumalo NP, Stein DJ (2018) Determinants of hair cortisol concentration in children: a systematic review. Psychoneuroendocrinology 87:204-214

81. Martin JM, Green M, Barbadora KA, Wald ER (2004) Group A streptococci among school-aged children: clinical characteristics and the carrier state. Pediatrics 114(5):1212-1219

82. McMahon WM, Carter AS, Fredine N, Pauls DL (2003) Children at familial risk for Tourette's disorder: child and parent diagnoses. Am J Med Genet B Neuropsychiatr Genet 121(1):105-111
83. Spinello C, Laviola G, Macri S (2016) Pediatric autoimmune disorders associated with streptococcal infections and Tourette's syndrome in preclinical studies. Front Neurosci 10:310

84. Macri S, Proietti OM, Laviola G (2013) Theoretical and practical considerations behind the use of laboratory animals for the study of Tourette syndrome. Neurosci Biobehav Rev 37(6):1085-1100

85. Macri S, Onori MP, Roessner V, Laviola G (2013) Animal models recapitulating the multifactorial origin of Tourette syndrome. Int Rev Neurobiol 112:211-237

86. Tang Y, Schapiro MB, Franz DN, Patterson BJ, Hickey FJ, Schorry EK et al (2004) Blood expression profiles for tuberous sclerosis complex 2, neurofibromatosis type 1, and Down's syndrome. Ann Neurol 56(6):808-814

87. Borovecki F, Lovrecic L, Zhou J, Jeong H, Then F, Rosas HD et al (2005) Genome-wide expression profiling of human blood reveals biomarkers for Huntington's disease. Proc Natl Acad Sci USA 102(31):11023-11028

88. Achiron A, Gurevich M, Friedman N, Kaminski N, Mandel M (2004) Blood transcriptional signatures of multiple sclerosis: unique gene expression of disease activity. Ann Neurol 55(3):410-417

89. Du X, Tang Y, Xu H, Lit L, Walker W, Ashwood P et al (2006) Genomic profiles for human peripheral blood T cells, B cells, natural killer cells, monocytes, and polymorphonuclear cells: comparisons to ischemic stroke, migraine, and Tourette syndrome. Genomics 87(6):693-703

90. Lit L, Gilbert DL, Walker W, Sharp FR (2007) A subgroup of Tourette's patients overexpress specific natural killer cell genes in blood: a preliminary report. Am J Med Genet B Neuropsychiatr Genet 144B(7):958-963

91. Liao IH, Corbett BA, Gilbert DL, Bunge SA, Sharp FR (2010) Blood gene expression correlated with tic severity in medicated and unmedicated patients with Tourette Syndrome. Pharmacogenomics 11(12): 1733-1741

92. Tian Y, Stamova B, Ander BP, Jickling GC, Gunther JR, Corbett BA et al (2012) Correlations of gene expression with ratings of inattention and hyperactivity/impulsivity in Tourette syndrome: a pilot study. BMC Med Genomics 30(5):49

\section{Affiliations}

\section{Anette Schrag ${ }^{1} \cdot$ Davide Martino $^{2} \cdot$ Alan Apter $^{3} \cdot$ Juliane Ball $^{4} \cdot$ Erika Bartolini $^{5} \cdot$ Noa Benaroya-Milshtein $^{3}$. Maura Buttiglione $^{6} \cdot$ Francesco Cardona $^{7} \cdot$ Roberta Creti $^{8} \cdot$ Androulla Efstratiou $^{9} \cdot$ Maria Gariup $^{10,11}$.

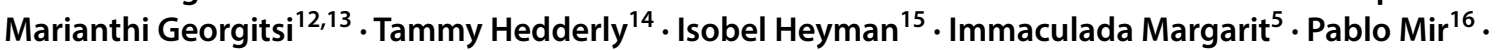 Natalie Moll ${ }^{17}$. Astrid Morer ${ }^{18,19,20}$. Norbert Müller ${ }^{21,22} \cdot$ Kirsten Müller-Vahl $^{23}$. Alexander Münchau ${ }^{24}$. ${\text { Graziella Orefici }{ }^{8} \cdot \text { Kerstin J. Plessen }}^{25,26} \cdot$ Cesare Porcelli $^{27} \cdot$ Peristera Paschou ${ }^{28} \cdot$ Renata Rizzo $^{29} \cdot$ Veit Roessner $^{30}$. Markus J. Schwarz ${ }^{17}$. Tamar Steinberg ${ }^{3} \cdot$ Friederike Tagwerker Gloor $^{4} \cdot$ Zsanett Tarnok $^{31} \cdot$ Susanne Walitza ${ }^{4}$. Andrea Dietrich $^{32}$. Pieter J. Hoekstra ${ }^{32} \cdot$ EMTICS Collaborative Group}

1 Department of Clinical Neurosciences, UCL Institute of Neurology, University College London, London, UK

2 Department of Clinical Neurosciences, University of Calgary, Calgary, Canada

3 Child and Adolescent Psychiatry Department, Schneider Children's Medical Center of Israel, Affiliated to Sackler Faculty of Medicine, Tel Aviv University, Petah-Tikva, Israel

4 Clinic of Child and Adolescent Psychiatry and Psychotherapy, University of Zurich, Zurich, Switzerland
5 GSK, Siena, Italy

6 Department of Biological Sciences and Human Oncology, Medical School, University of Bari "Aldo Moro", Bari, Italy

7 Department of Human Neurosciences, University La Sapienza of Rome, Rome, Italy

8 Department of Infectious Diseases, Istituto Superiore di Sanità, Rome, Italy

9 WHO Global Collaborating Centre for Reference and Research on Diphtheria and Streptococcal Infections, 
Reference Microbiology, Directorate National Infection Service, Public Health England, London, UK

10 University of Barcelona, Barcelona, Spain

11 Intensive Inpatient Unit, Copenhagen Psychiatric Center, Copenhagen, Denmark

12 Department of Molecular Biology and Genetics, Democritus University of Thrace, Alexandroupoli, Greece

13 Department of Medicine, Aristotle University of Thessaloniki, Thessaloníki, Greece

14 Evelina London Children's Hospital GSTT, Kings Health Partners AHSC, London, UK

15 Great Ormond Street Hospital for Children, UCL Institute of Child Health, London, UK

16 Unidad de Trastornos del Movimiento, Servicio de Neurología y Neurofisiología Clinica, Instituto de Biomedicina de Sevilla (IBiS), Hospital Universitario Virgen del Rocio/CSIC/Universidad de Sevilla, Seville, Spain

17 Institute of Laboratory Medicine, University Hospital, LMU Munich, Munich, Germany

18 Department of Child and Adolescent Psychiatry and Psychology, Institute of Neurosciences, Hospital Clinic Universitari, Barcelona, Spain

19 Institut d'Investigacions Biomediques August Pi i Sunyer (IDIBAPS), Barcelona, Spain

20 Centro de Investigacion en Red de Salud Mental (CIBERSAM), Instituto Carlos III, Madrid, Spain

21 Department of Psychiatry and Psychotherapy, University Hospital, LMU Munich, Munich, Germany
Marion von Tessin Memory-Zentrum gGmbH, Munich, Germany

23

Clinic of Psychiatry, Social Psychiatry and Psychotherapy, Hannover Medical School, Hannover, Germany

24 Institute of Neurogenetics, University of Lübeck, Lübeck, Germany

25 Child and Adolescent Mental Health Center, Mental Health Services, Capital Region of Denmark and University of Copenhagen, Copenhagen, Denmark

26 Service of Child and Adolescent Psychiatry, Department of Psychiatry, University Medical Center, University of Lausanne, Lausanne, Switzerland

27 Azienda Sanitaria Locale di Bari, Mental Health Department, Child and Adolescent Neuropsychiatry Service of Bari Metropolitan Area, Bari, Italy

28 Department of Biological Sciences, Purdue University, West Lafayette, USA

29 Child Neuropsychiatry Section, Department of Clinical and Experimental Medicine, School of Medicine, Catania University, Catania, Italy

30 Department of Child and Adolescent Psychiatry, Faculty of Medicine, TU Dresden, Dresden, Germany

31 Vadaskert Child and Adolescent Psychiatric Hospital, Budapest, Hungary

32 Department of Child and Adolescent Psychiatry, University Medical Center Groningen, University of Groningen, Hanzeplein 1, 9713 GZ Groningen, The Netherlands 